\title{
Ranking the in vivo toxicity of nanomaterials in Drosophila melanogaster
}

\author{
G. Vecchio • A. Galeone - M. A. Malvindi • \\ R. Cingolani $\cdot$ P. P. Pompa
}

Received: 3 October 2012/ Accepted: 12 August 2013

(C) Springer Science+Business Media Dordrecht 2013

\begin{abstract}
In this work, we propose a quantitative assessment of nanoparticles toxicity in vivo. We show a quantitative ranking of several types of nanoparticles (AuNPs, AgNPs, cadmium-based QDs, cadmium-free QDs, and iron oxide NPs, with different coating and/or surface chemistries), providing a categorization of their toxicity outcomes. This strategy may offer an innovative high-throughput screening tool of nanomaterials, of potential and broad interest to the nanoscience community.
\end{abstract}

Special Issue Editors: Juan Manuel Rojo, Vasileios Koutsos

This article is part of the topical collection on Nanostructured Materials 2012

G. Vecchio and A. Galeone have contributed equally to this study.

Electronic supplementary material The online version of this article (doi:10.1007/s11051-013-1936-3) contains supplementary material, which is available to authorized users.

G. Vecchio - A. Galeone - M. A. Malvindi .

P. P. Pompa ( $\square)$

Center for Bio-Molecular Nanotechnologies@UniLe, Istituto Italiano di Tecnologia (IIT), Via Barsanti, 1,

73010 Arnesano, LE, Italy

e-mail: pierpaolo.pompa@iit.it

R. Cingolani

Central Research Laboratories, Istituto Italiano di

Tecnologia (IIT), Via Morego, 30, 16136 Genoa, Italy
Keywords Nanoparticles · Nanotoxicology · In vivo $\cdot$ Quantitative ranking

For many years, the nanoscience and nanotechnology community attempted to design and develop innovative processes and materials with novel/enhanced properties, but only in the early 2000s some nanoproducts started to have commercial relevance. During the latest years, the production of nanomaterials (NMs) increased rapidly, as well as their use in various fields, especially in biomedicine and biotechnology. Simultaneously, the awareness of the possible impact of NMs on human health and environment and the consequent necessity to regulate their commercial use are growing, posing urgent questions about their potential toxicity. As a result, a new area of toxicology, named nanotoxicology, a discipline that studies the toxic effects of NMs in biological systems and the interactions occurring between NMs and biomolecules/biological fluids, is rapidly expanding, evidencing some potential risks related to their indiscriminate use. The huge efforts performed by the scientific community and funding agencies recently succeeded in providing several key findings and important observations in nanotoxicology (Maynard et al. 2011; Warheit 2008; Nature Nanotechnology, Focus Nanotoxicology 2012), partially clarifying some unknown aspects of this new science. However, many issues concerning the potential toxicity of NMs remain largely unclear. The primary difficulties are mainly 
related to the huge variety of NMs, each characterized by a peculiar set of physico-chemical properties. Unlike classic toxicological studies, in fact, nanotoxicology investigations require not only information about the administration dose, but also detailed analyses about the material under test, such as size, shape, surface chemistry, reactivity, composition, dispersion status, stability, etc., as all such parameters were demonstrated to play fundamental roles in the biological interactions and related toxicity (Krug and Wick 2011; Oberdörster 2010; Oberdorster et al. 2005). Furthermore, biological matrix and fluids, in which NMs are dispersed, should be carefully considered in order to evaluate the nanotoxicity effects. In particular, the interactions with biomolecules may strongly influence the stability, dispersion, and size of NMs and, in turn, the cellular uptake (i.e., the dose) and toxicity outcome, so the in situ characterization of NPs has been recently recognized to be fundamental (Haniu et al. 2011; Kim et al. 2011; Lynch and Dawson 2008; Monopoli et al. 2012; Maiorano et al. 2010; Dawson et al. 2012). Another crucial aspect is the need of massive screening of the numerous NMs, with the aim of obtaining precise and reliable assessment of their toxic effects, possibly achieving quantitative data. This would be of great interest in the perspective to develop worldwide approved regulation, establishing risk assessment procedures and general rules for their correct use.

In this work, we propose a quantitative ranking of the in vivo toxicity of several types of NMs, trying to categorize their biological outcomes. We focused our attention on the ingestion route of NMs. To this purpose, we used Drosophila melanogaster, a wellestablished model in toxicology (Rand 2010) and nanotoxicology (Ahamed et al. 2010; Galeone et al. 2012; Pompa et al. 2011a; Vecchio et al. 2012a, b; Liu et al. 2009; Vales et al. 2013). Drosophila represents an effective model organism to investigate the molecular mechanisms of human diseases because it shares with mammals many basic biological, physiological, and neurological properties, and it is also considered a valid tool for therapeutic discovery. Furthermore, Drosophila has some equivalent functions of the mammalian organs (heart, lung, kidney, gut, and reproductive tract) and possesses similar basic metabolic mechanisms that make adult fly a very sophisticated and complex organism not unlike higher organisms (Pandey and Nichols 2011; Wang et al.
2012). Finally, although there are many differences between flies and humans, the degree of conserved biology and physiology makes Drosophila an extremely valuable tool for toxicological pre-screening of NMs administered by ingestion, thanks to its short lifespan and well-characterized development. This enables systematic screening of many NMs in relatively short time, reduced costs, and limited ethical issues, in agreement with the principles of replacement, reduction, and refinement (known as the 3Rs) that aim to reduce the impact of scientific activities on mammalian animals (Flecknell 2002). We analyzed different types of largely employed engineered nanoparticles: gold nanoparticles (AuNPs) with different surface chemistries, silver nanoparticles (AgNPs), three differently coated cadmium-based QDs (CdSe/ $\mathrm{ZnS}$ ), cadmium-free QDs (InP/ZnS), and two differently surface passivated iron oxide $\left(\mathrm{Fe}_{3} \mathrm{O}_{4}\right)$ NPs. These NPs were characterized by dynamic light scattering (DLS), TEM, and Z-pot (see Table 1) and showed to be highly monodispersed and stable in aqueous solutions. Moreover, we also performed the in situ characterization of the NMs (namely, upon dispersion in the Drosophila food) by TEM analyses, finding that they basically maintain their original size and monodispersion (Supplementary material Fig. S1).

To assess the possible toxic effects of these NMs, we administered different concentrations of each NPs (ranging from $50 \mathrm{pM}$ to $1 \mathrm{nM}$ ) to D. melanogaster by ingestion, for their entire lifespan. The toxic effects of the different NPs were evaluated in terms of lifespan reduction of the organisms, as compared to the control

Table 1 Physico-chemical characterizations of NPs by DLS, TEM, and Z-pot

\begin{tabular}{lrrr}
\hline Nanoparticles & \multicolumn{1}{c}{ DLS $(\mathrm{nm})$} & TEM $(\mathrm{nm})$ & Z-pot $(\mathrm{mV})$ \\
\hline AuNPs & $17.7 \pm 2.2$ & $15.2 \pm 0.9$ & $-38.3 \pm 5.2$ \\
AuNPs-PEG & $24 \pm 3.0$ & $15.2 \pm 0.9$ & $-16.0 \pm 4.0$ \\
$\mathrm{InP} / Z n S Q D s$ & $11.34 \pm 0.6$ & $2.7 \pm 0.7$ & $-26.0 \pm 9.0$ \\
$\mathrm{Fe}_{3} \mathrm{O}_{4} \mathrm{NPs}$ & $28.0 \pm 5.0$ & $25.0 \pm 3.0$ & $-33.0 \pm 5.0$ \\
$\mathrm{Fe}_{3} \mathrm{O}_{4} \mathrm{NPs}$ (Funct) & $28.0 \pm 5.0$ & $25.0 \pm 2.0$ & $-46.0 \pm 7.0$ \\
$\mathrm{CdSe} / \mathrm{ZnS}$ QDs & $15.0 \pm 1.5$ & $6.0 \pm 1.0$ & $-29.0 \pm 3.0$ \\
$\mathrm{CdSe} / \mathrm{ZnS}$ QDs-PC & $23.0 \pm 1.6$ & $6.0 \pm 0.5$ & $-30.0 \pm 2.0$ \\
$\mathrm{CdSe} / \mathrm{ZnSQDs}-$ & $25.0 \pm 1.5$ & $7.0 \pm 0.8$ & $-25.0 \pm 1.4$ \\
$\quad \mathrm{PC}-\mathrm{PEG}$ & & & \\
AgNPs & $6.0 \pm 2.1$ & $5.5 \pm 2.0$ & $-25.6 \pm 3.1$ \\
\hline
\end{tabular}


untreated animals. We also evaluated the effects of the solutions in which the NMs were dispersed, finding no detectable toxicity (the results were comparable to the controls).

Figure 1 reports the dose-dependent toxicity response of the different NPs, expressed as the fractional reduction of the organism lifespan with respect to the control represented by the Drosophila nurtured with normal food (some representative points were chosen for each NPs type). The black line represents the dose-response curve obtained with 15-nm citrate-capped AuNPs (experimental data were fitted by a bi-exponential decay curve) (Pompa et al. 2011b). We used AuNPs as the reference curve because the toxic effects induced by this well-characterized NM are noticeable but lower than that of other types of nanoparticles (e.g., AgNPs), when administered to Drosophila at the same concentrations. In fact, even at high AuNP doses, the lifespan of Drosophila is ca. $60 \%$ compared to the untreated control flies, representing a kind of threshold between highly toxic and low/medium toxic materials. Below such reference curve, the toxic effects become so severe to induce significant mortality in treated organisms and consequent dramatic reduction of their average lifespan.

As shown, cadmium-based QDs, iron oxide NPs, and AgNPs exhibited a very strong lifespan reduction, falling in the red region of the toxicity space, and being classified as highly toxic NMs. Among these, AgNPs

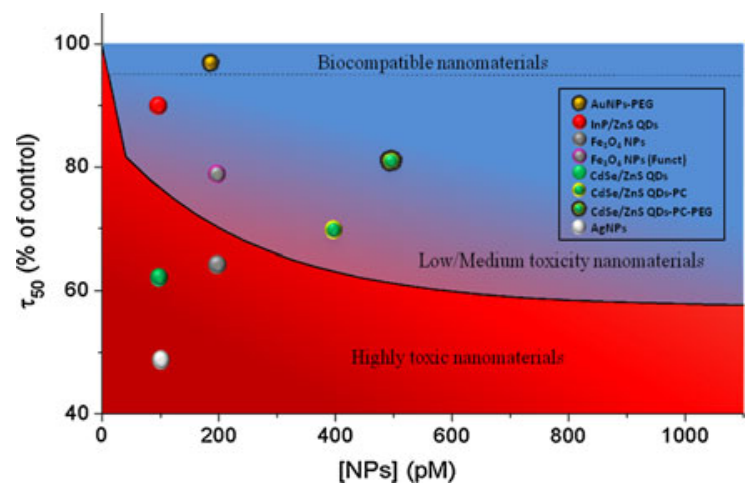

Fig. 1 Schematic picture representing the universal toxicity curve based on the reference bi-exponential fit of the citratecapped AuNPs (Pompa et al. 2011b). Representative toxicity levels of AuNPs-PEG (200 pM), InP/ZnS QDs (100 pM), $\mathrm{Fe}_{3} \mathrm{O}_{4}$ NPs $(200 \mathrm{pM}), \mathrm{Fe}_{3} \mathrm{O}_{4}$ NPs (Funct) $(200 \mathrm{pM}), \mathrm{CdSe} / \mathrm{ZnS}$ QDs (100 pM), CdSe/ZnS QDs-PC (400 pM), CdSe/ZnS QDs-PCPEG (500 pM), and AgNPs are shown are the most toxic upon ingestion, exhibiting a lifespan decrease of ca. $50 \%$ with respect to the control, even at very low concentrations ( $100 \mathrm{pM})$. This finding is fairly consistent with the literature, as many in vitro and in vivo studies have recently demonstrated significant toxicity of AgNPs (Arora et al. 2008; van der Zande et al. 2012). In general, depending on size, shape, surface chemistry, solubility, and stability, the toxic effects of AgNPs are mainly known to induce high increase in ROS levels, leading to cell apoptosis by impairment of mitochondrial activity. The detailed mechanism of AgNPs-induced toxicity remains unclear, although the NPs surface area and the release of free silver ions have been reported to play important roles. Notably, silver ions may have detrimental effect on biological systems, causing oxidative stress, as well as DNA, protein, and lipid damage (Arora et al. 2008; van der Zande et al. 2012).

Concerning cadmium QDs, the lifespan reduction seems to be closely related to the surface coatings of the NPs (Fig. 1). In particular, the CdSe QDs are highly toxic, showing a strong lifespan decrease (ca. $40 \%$ reduction with respect to the control, at a concentration as low as $100 \mathrm{pM})$. On the other hand, the viability decrease of coated CdSe QDs (QDs-PC and QDs-PC-PEG) is more attenuated (ca. $30 \%$ reduction at $400 \mathrm{pM}$ and $20 \%$ at $500 \mathrm{pM}$, respectively). The toxicity of these NMs can be mainly ascribed to their degradation in biological systems with the consequent release of cadmium ions, in turn eliciting significant increase of oxidative stress and induction of cellular homeostasis response (Galeone et al. 2012; Chen et al. 2012). The surface engineering of QDs (by polymer and PEG functionalization) attenuates the lifespan decrease (Fig. 1), mainly because it reduces the uptake/bioaccumulation of QDs in the organism, and so the overall toxicity (Galeone et al. 2012).

The $\mathrm{Fe}_{3} \mathrm{O}_{4} \mathrm{NPs}$, falling in the red region, can be also classified as highly toxic, displaying ca. $30 \%$ of viability reduction at low concentration $(200 \mathrm{pM})$. In this case, an excessive bioaccumulation of iron oxide NPs can overload cells and tissues with metal ions. This can lead to an imbalance in cell homeostasis and can cause aberrant cellular responses, including cytotoxicity, DNA damage, oxidative stress, epigenetic events, and inflammatory processes (Singh et al. 2010). On the other hand, when the surface of iron oxide NPs is passivated by chemical functionalization 
$\left(\mathrm{Fe}_{3} \mathrm{O}_{4} \mathrm{NPs}\right.$ (Funct)) in order to decrease the release of ions in vivo, the overall toxicity was found to be reduced, though not completely eliminated (ca. $20 \%$ viability decrease compared to the control) at the same concentration $(200 \mathrm{pM})$.

In the experiments with cadmium-free NPs (InP QDs), a slight lifespan reduction (ca. $10 \%$ at $100 \mathrm{pM}$ ) was detected, indicating low toxicity in Drosophila. These nanoparticles have similar size and surface chemistry compared to CdSe QDs. Hence, the much lower toxicity observed in vivo is most likely due to the replacement of the highly toxic cadmium with the more tolerated indium (Chibli et al. 2011). In fact, although we assessed that InP QDs also tend to degrade in in vivo conditions, releasing indium ions, such metal does not significantly impact cellular environments. These experimental data suggest that InP QDs, keeping similar optical characteristics compared to cadmium-based QDs, may be a promising alternative for biological and biomedical applications.

Finally, pegylated AuNPs (AuNPs-PEG) showed good biocompatibility, exhibiting a very low lifespan reduction $(<5 \%$ at $200 \mathrm{pM})$, so we classified them as non-toxic NMs. As reported by several in vitro and in vivo studies, pegylation significantly decreases the cellular uptake and bioaccumulation of NPs (Arnida et al. 2010; Galeone et al. 2012). In line with such observations, we thus found a reduced bioaccumulation of AuNPs-PEG in the flies and, consequently, a largely reduced toxicity (as compared to citratecapped AuNPs). Importantly, such surface modification is highly relevant in the case of AuNPs, as we recently observed that naked nanogold can elicit remarkable toxicity both in vitro and in vivo (Sabella et al. 2011), as well as genotoxicity and mutagenicity (Vecchio et al. 2012a).

In addition to this qualitative, "visual" assessment, using the different regions in the toxicity space (Fig. 1), we tried to define a quantitative ranking of the in vivo toxicity of these NMs, through the toxicity factor (Pompa et al. 2011b). The experimental half-life $\left(\tau_{50} \%\right)$ data of the Drosophila populations treated with the different NPs were fitted by a bi-exponential decay curve. Then, for each NP type, we calculated the "toxicity factor," defined as the slope of the viability decay curve in the low concentration range, namely $y^{\prime}(x \rightarrow 0)$ (Pompa et al. 2011b). For each NM, the calculated toxicity factor is reported in Fig. 2. As shown, the toxicity factor is more negative in the case of highly toxic NPs, such as AgNPs and cadmiumbased QDs (ca. -38 and -27 , respectively), while it approaches less negative values for less toxic NMs, e.g., pegylated AuNPs (toxicity factor: ca. -1.5). By this methodology, it is thus possible to "quantify" the toxicity of a specific NPs, by attributing it a number that expresses its toxicity.

Based on the above results, we have categorized the toxic effects of the investigated NMs in three main groups:

(i) Highly toxic NMs: AgNPs, cadmium-based QDs, and iron oxide NPs. All these NPs strongly affected Drosophila lifespan, exhibiting a very negative toxicity factor.

(ii) Medium toxic NMs: coated Cd QDs (QDs-PC and QDs-PC-PEG), and surface-passivated iron oxide NPs.

(iii) Biocompatible or nearly biocompatible NMs: AuNPs-PEG and InP QDs.

In conclusion, in this work we have proposed a quantitative methodology to evaluate NMs toxicity in vivo, using a physical quantification through the toxicity factor. This approach may provide a powerful high-throughput tool to strengthen nanotoxicology investigations in vivo, predicting the toxicity outcomes of different NMs. This may be important for a wide range of nanomedicine applications, as well as for the development of a reliable risk assessment and regulatory approval. On the other side, it should be mentioned that the proposed toxicity classification is based on the observed lifespan reduction elicited by the NMs administered by ingestion, while other important events, such as inflammation, and effects



Fig. 2 Ranking of the in vivo toxicity of NPs based on the toxicity factor 
related to different administration routes are not directly evaluated by this model. More accurate and refined screening tools for nanotoxicity assessment are thus required in the next years.

\section{Methods}

Drosophila melanogaster strain and culture conditions

The flies and larvae of wild-type D. melanogaster (Oregon $\mathrm{R}+$ ) were cultured at $24 \pm 1{ }^{\circ} \mathrm{C}$ on standard Drosophila food, containing agar, corn meal, sugar, yeast, and nepagin (methyl-p-hydroxybenzoate).

\section{NMs exposure and Drosophila lifespan studies}

NMs were formulated in the Drosophila diet with concentration ranging from $50 \mathrm{pM}$ to $1 \mathrm{nM}$. Such concentrations correspond to the following NM doses: AuNPs-PEG (from 1.54 to $30.75 \mu \mathrm{g} / \mathrm{g}$ per day); InP/ $\mathrm{ZnS}$ QDs (from $0.86 \mathrm{ng} / \mathrm{g}$ to $15 \mu \mathrm{g} / \mathrm{g}$ per day); $\mathrm{Fe}_{3} \mathrm{O}_{4}$ NPs (Funct), and $\mathrm{Fe}_{3} \mathrm{O}_{4}$ NPs (from 1.91 to $38.25 \mu \mathrm{g} / \mathrm{g}$ per day); CdSe/ZnS QDs, CdSe/ZnS QDs-PC, and CdSe/ZnS QDs-PC-PEG (from $5 \mathrm{ng} / \mathrm{g}$ to $0.1 \mu \mathrm{g} / \mathrm{g}$ per day); and AgNPs (from $37.5 \mathrm{ng} / \mathrm{g}$ to $0.75 \mu \mathrm{g} / \mathrm{g}$ per day). All the stocks of NMs used in this work consist of aqueous solutions, and were diluted in ultrapure water prior to the mixing with Drosophila food. The diluted solutions containing the NMs were added to the food before solidification, mixed strongly, and finally poured into vials. Lifespan experiments were performed as previously reported (Vecchio et al. 2012b). Briefly, for longevity analyses, newly eclosed flies were collected and housed at a density of 20 males and 20 females, separately, per each vial. Flies were transferred into fresh food every 3-4 days, and dead flies were counted every day until all died. We carried out this experiment using normal food and treated food containing different types of NMs.

\section{NMs synthesis}

Silver nanoparticles (AgNPs) were synthesized by photochemical method by using tyrosine as a photoreducing agent (Kshirsagar et al. 2011). The synthesis was carried out in a laboratory reactor system fitted with UV lamp and surrounded by quartz tubing for cooling with water. The pre-cooled aqueous solution of potassium hydroxide irradiated with the UV lamp was added with tyrosine and silver ions $\left(\mathrm{Ag}_{2} \mathrm{SO}_{4}\right)$ under vigorous stirring conditions. After $30 \mathrm{~min}$, the AgNPs solution was warmed up to room temperature. The concentrated NPs suspension was purified and separated by using Sephadex G-75.

For CdSe/ZnS QDs, TOP/TOPO-capped QDs were prepared by following the standard colloidal synthesis procedures (Galeone et al. 2012). The as-synthesized nanocrystals were transferred from the organic phase to aqueous phase by exchanging the surface ligands to 11-mercaptoundecanoic acid (CdSe QDs) and by adopting a polymer-coating procedure (CdSe QDsPC, CdSe QD-PC-PEG) (Di Corato et al. 2008; Pellegrino et al. 2004).

Spherical $\mathrm{Fe}_{3} \mathrm{O}_{4}$ NPs were synthesized by a modified surfactant-assisted nonaqueous synthetic approach (Hyeon et al. 2001). The nanoparticles were subsequently transferred into water, by coating their surface with a silica shell using an inverse microemulsion method (Vogt et al. 2010). Some of these NPs were further surface passivated by 3-(trihydroxysilyl)-1-propanesulfonic acid (SIT) to reduce ion leakage.

Citrate-capped AuNPs were prepared as previously reported (Pompa et al. 2011b; Vecchio et al. 2012a). For PEGylation of AuNPs (AuNPs-PEG), NPs were modified using thiol-terminated polyethylene glycol (HS-PEG, of MW 5000).

InP/ZnS QDs (InP QDs) were synthesized using a method adapted from the literature (Chibli et al. 2011) with slight variation. To transfer the InP QDs into aqueous environment, butanol, borate buffer at $\mathrm{pH} 9$, and mercaptopropionic acid were added to the InP QDs. The mixture was heated at $50{ }^{\circ} \mathrm{C}$ for $15 \mathrm{~min}$. The two phases were separated and the solution containing the InP QDs was purified by washing/filtering four times using a 10,000 molecular weight cutoff filter.

\section{NMs characterization}

For TEM analyses, all samples were prepared by dropping a dilute solution of NPs in water on carboncoated copper grids (Formvar/Carbon 300 Mesh Cu). TEM images were recorded on a JEOL Jem1011 
microscope operating at an accelerating voltage of $100 \mathrm{kV}$. DLS and Z-potential measurements analysis were carried out using Zetasizer Nano ZS90 (Malvern, USA) equipped with a 4.0-mW He-Ne laser operating at $633 \mathrm{~nm}$ and an avalanche photodiode detector. Measurements were made at $25{ }^{\circ} \mathrm{C}$ in water, $\mathrm{pH} 7$. Each sample was measured five times and the results were analyzed by Malvern Instruments Ltd software.

Acknowledgments The authors gratefully acknowledge P. Kshirsagar, G. Maiorano, H. Chibli, and V. De Matteis for NPs synthesis and characterization and help during experiments, and V. Fiorelli and B. Antonazzo for the expert technical assistance.

\section{References}

Ahamed M, Posgai R, Gorey TJ, Nielsen M, Hussain SM, Rowe JJ (2010) Silver nanoparticles induced heat shock protein 70 , oxidative stress and apoptosis in Drosophila melanogaster. Toxicol Appl Pharmacol 242(3):263-269

Arnida, Malugin A, Ghandehari H (2010) Cellular uptake and toxicity of gold nanoparticles in prostate cancer cells: a comparative study of rods and spheres. J Appl Toxicol 30(3):212-217

Arora S, Jain J, Rajwade JM, Paknikar KM (2008) Cellular responses induced by silver nanoparticles: in vitro studies. Toxicol Lett 179(2):93-100

Chen N, He Y, Su Y, Li X, Huang Q, Wang H, Zhang X, Tai R, Fan C (2012) The cytotoxicity of cadmium-based quantum dots. Biomaterials 33(5):1238-1244

Chibli H, Carlini L, Park S, Dimitrijevic NM, Nadeau JL (2011) Cytotoxicity of InP/ZnS quantum dots related to reactive oxygen species generation. Nanoscale 3(6):2552-2559

Dawson KA, Anguissola S, Lynch I (2012) The need for in situ characterisation in nanosafety assessment: funded transnational access via the QNano research infrastructure. Nanotoxicology. doi:10.3109/17435390.2012.658096

Di Corato R, Quarta A, Piacenza P, Ragusa A, Figuerola A, Buonsanti R, Cingolani R, Manna L, Pellegrino T (2008) Water solubilization of hydrophobic nanocrystals by means of poly(maleic anhydride-alt-1-octadecene). J Mater Chem 18(17):1991-1996

Flecknell P (2002) Replacement, reduction and refinement. ALTEX 19(2):73-78

Galeone A, Vecchio G, Malvindi MA, Brunetti V, Cingolani R, Pompa PP (2012) In vivo assessment of CdSe-ZnS quantum dots: coating dependent bioaccumulation and genotoxicity. Nanoscale 4(20):6401-6407

Haniu H, Saito N, Matsuda Y, Kim YA, Park KC, Tsukahara T, Usui Y, Aoki K, Shimizu M, Ogihara N, Hara K, Takanashi S, Okamoto M, Ishigaki N, Nakamura K, Kato H (2011) Effect of dispersants of multi-walled carbon nanotubes on cellular uptake and biological responses. Int J Nanomed 6:3295-3307

Hyeon T, Lee SS, Park J, Chung Y, Na HB (2001) Synthesis of highly crystalline and monodisperse maghemite nanocrystallites without a size-selection process. J Am Chem Soc 123(51):12798-12801

Kim J, Song K, Lee J, Yu I (2011) Evaluation of biocompatible dispersants for carbon nanotube toxicity tests. Arch Toxicol 85(12):1499-1508

Krug HF, Wick P (2011) Nanotoxicology: an interdisciplinary challenge. Angew Chemie Int Ed 50(6):1260-1278

Kshirsagar P, Sangaru SS, Malvindi MA, Martiradonna L, Cingolani R, Pompa PP (2011) Synthesis of highly stable silver nanoparticles by photoreduction and their size fractionation by phase transfer method. Colloids Surf A 392(1):264-270

Liu X, Vinson D, Abt D, Hurt RH, Rand DM (2009) Differential toxicity of carbon nanomaterials in Drosophila: larval dietary uptake is benign, but adult exposure causes locomotor impairment and mortality. Environ Sci Technol 43(16):6357-6363

Lynch I, Dawson KA (2008) Protein-nanoparticle interactions. Nano Today 3(1-2):40-47

Maiorano G, Sabella S, Sorce B, Brunetti V, Malvindi MA, Cingolani R, Pompa PP (2010) Effects of cell culture media on the dynamic formation of protein-nanoparticle complexes and influence on the cellular response. ACS Nano 4(12):7481-7491

Maynard AD, Warheit DB, Philbert MA (2011) The new toxicology of sophisticated materials: nanotoxicology and beyond. Toxicol Sci 120(Suppl 1):S109-S129

Monopoli MP, Aberg C, Salvati A, Dawson KA (2012) Biomolecular coronas provide the biological identity of nanosized materials. Nat Nanotechnol 7(12):779-786

Nature Nanotechnology, Focus Nanotoxicology (2012) http:// www.nature.com/nnano/focus/nanotoxicology/index.html. Accessed 2012

Oberdörster G (2010) Safety assessment for nanotechnology and nanomedicine: concepts of nanotoxicology. J Int Med 267(1):89-105

Oberdorster G, Oberdorster E, Oberdorster J (2005) Nanotoxicology: an emerging discipline evolving from studies of ultrafine particles. Environ Health Perspect 113(7):823-839

Pandey UB, Nichols CD (2011) Human disease models in Drosophila melanogaster and the role of the fly in therapeutic drug discovery. Pharmacol Rev 63(2):411-436

Pellegrino T, Manna L, Kudera S, Liedl T, Koktysh D, Rogach AL, Keller S, Rädler J, Natile G, Parak WJ (2004) Hydrophobic nanocrystals coated with an amphiphilic polymer shell: a general route to water soluble nanocrystals. Nano Lett 4(4):703-707

Pompa P, Vecchio G, Galeone A, Brunetti V, Sabella S, Maiorano G, Falqui A, Bertoni G, Cingolani R (2011a) In vivo toxicity assessment of gold nanoparticles in Drosophila melanogaster. Nano Res 4(4):405-413

Pompa PP, Vecchio G, Galeone A, Brunetti V, Maiorano G, Sabella S, Cingolani R (2011b) Physical assessment of toxicology at nanoscale: nano dose-metrics and toxicity factor. Nanoscale 3(7):2889-2897

Rand MD (2010) Drosophotoxicology: the growing potential for Drosophila in neurotoxicology. Neurotoxicol Teratol 32(1):74-83

Sabella S, Brunetti V, Vecchio G, Galeone A, Maiorano G, Cingolani R, Pompa P (2011) Toxicity of citrate-capped 
AuNPs: an in vitro and in vivo assessment. J Nanopart Res 13(12):6821-6835

Singh N, Jenkins GJ, Asadi R, Doak SH (2010) Potential toxicity of superparamagnetic iron oxide nanoparticles (SPION). Nano Rev. doi:10.3402/nano.v1i0.5358

Vales G, Demir E, Kaya B, Creus A, Marcos R (2013) Genotoxicity of cobalt nanoparticles and ions in Drosophila. Nanotoxicology 7(4):462-468

van der Zande M, Vandebriel RJ, Van Doren E, Kramer E, Herrera Rivera Z, Serrano-Rojero CS, Gremmer ER, Mast J, Peters RJB, Hollman PCH, Hendriksen PJM, Marvin HJP, Peijnenburg AACM, Bouwmeester H (2012) Distribution, elimination, and toxicity of silver nanoparticles and silver ions in rats after 28-day oral exposure. ACS Nano 6(8):7427-7442

Vecchio G, Galeone A, Brunetti V, Maiorano G, Rizzello L, Sabella S, Cingolani R, Pompa PP (2012a) Mutagenic effects of gold nanoparticles induce aberrant phenotypes in Drosophila melanogaster. Nanomedicine NBM 8(1):1-7
Vecchio G, Galeone A, Brunetti V, Maiorano G, Sabella S, Cingolani R, Pompa PP (2012b) Concentration-dependent, size-independent toxicity of citrate capped AuNPs in Drosophila melanogaster. PLoS ONE 7(1):e29980

Vogt C, Toprak M, Muhammed M, Laurent S, Bridot J-L, Müller R (2010) High quality and tuneable silica shellmagnetic core nanoparticles. J Nanopart Res 12(4):11371147

Wang B, Chen N, Wei YL, Li J, Sun L, Wu JR, Huang Q, Liu C, Fan CH, Song HY (2012) Akt signaling-associated metabolic effects of dietary gold nanoparticles in Drosophila. Sci Rep 2:563

Warheit DB (2008) How meaningful are the results of nanotoxicity studies in the absence of adequate material characterization? Toxicol Sci 101(2):183-185 